\title{
Tutoría y orientación educativa: la otra cara del acompañamiento en la educación a distancia
}

Arturo de Jesús Madrigal Gil ${ }^{1}$

\begin{abstract}
Resumen
Resultado de dos investigaciones, una concluida sobre concepciones de libertad en jóvenes universitarios de primer semestre (Betancur et al., 2007) y otra en curso sobre innovaciones curriculares (Garavito, Madrigal \& Urrego, 2009), además de un estudio que revisa una propuesta de diseño instruccional para la virtualidad (Garavito et al., 2009), surge la presente reflexión sobre el papel del docente tutor como acompañante que va más allá de la relación académica y le requiere en términos de apoyo, a veces consejero, dinamizador y frecuentemente articulador de la opción profesional con los proyectos de vida de los estudiantes, impactando la elección vocacional y la existencia misma de los tutorados.
\end{abstract}

Palabras clave: virtualidad, tutoría, acompañamiento, orientación, educativa, autonomía.

\begin{abstract}
Outcome of two investigations, one concluded on conceptions of liberty in first semester college students (Betancur et al., 2007) and another
\end{abstract}

1 Psicólogo Social Comunitario egresado de la UNAD. Magister en Educación y Desarrollo Humano del CINDE - Universidad de Manizales. Docente investigador, coordinador del área pedagógica de la Facultad de Educación Física y miembro del Grupo COMAEFI del Politécnico Colombiano Jaime Isaza Cadavid en la ciudad de Medellín. 


\section{Arturo de Jesús Madrigal Gil}

Tutoría y orientación educativa:

la otra cara del acompañamiento en la educación a distancia, artículo de reflexión

about curriculum innovations (Garavito, Madrigal \& Urrego, 2009), and a study that reviewed a proposal instructional design for the virtuality (Garavito et al., 2009), comes this reflection on the role of the teacher or tutor as a companion that goes beyond the academic relationship and it requires in terms of support, sometimes adviser, promoter and often professional option articulator with life projects for students, impacting vocational choice and the very existence of the poles.

Keywords: virtuality, tutoring, coaching, counseling, education, autonomy.

Recibido: 29 de abril de 2010

Aceptado: 25 de julio de 2010

\section{lntroducción}

La acción tutorial ha traspasado el territorio impuesto tradicionalmente por la academia encerrada en el límite de la relación docente-aprendiz en el contexto de una asignatura específica, para llegar a vincular en redes significativas de socialización la apropiación de esta opción pedagógica, hoy convertida también en un espacio para revitalizar los proyectos y sueños personales de los estudiantes.

Esta consideración se desprende de los resultados de las investigaciones y la revisión antes mencionadas sobre concepciones de libertad, innovaciones curriculares y diseño instruccional para la virtualidad, al encontrarse explícita la necesidad de pensar el encuentro docente- aprendiz en una dinámica de mayor cercanía vital que coadyuve a la asunción del rol de estudiante en la vivencia de las nuevas experiencias que ofrece la educación superior en términos de habilitar para el uso y aprovechamiento de la libertad en cada contexto en particular. Así, la relación mediatizada por las Tecnologías de la información y la comunicación comienza a presentarse como una opción para el escenario de la orientación educativa.

\section{Metodología}

El trabajo investigativo sobre concepciones de libertad fue desarrollado en el marco de la investigación cualitativa con enfoque hermenéutico, partiendo de los relatos ofrecidos por estudiantes de primer semestre y utilizando la entrevista a profundidad para la recolección de información. Estos relatos fueron procesados posteriormente a través de una matriz de análisis categorial que, en un ejercicio interpretativo, arroja un conjunto de concepciones de libertad que son luego validadas con los estudiantes entrevistados. 
La investigación sobre innovaciones curriculares, desarrollada con metodología mixta (cualitativa y cuantitativa) y con enfoque histórico-hermenéutico, ha utilizado como técnicas de recolección de información la encuesta, la entrevista, la revisión documental y la observación específicamente a los procesos académicos del trabajo independiente, en lo que atañe al acompañamiento no presencial del estudiante.

El estudio realizado para el Ministerio de Educación Nacional (MEN) en 2009, con el fin de elaborar el marco de referencia y la propuesta de trabajo para pasar cursos de la modalidad "a distancia" a la "virtual", exigió una revisión documental de la metodología a distancia y de los requerimientos para el tránsito de una modalidad a otra, además de las diferentes propuestas de diseño instruccional para la virtualidad. Allí fueron particularmente productivos los grupos de discusión donde, bajo la asesoría del Ministerio de Educación Nacional, se presentaban las diversas propuestas hasta llegar al diseño final que orientaría la elaboración de los cursos para los nuevos programas que se pretende ofrecer en esta modalidad.

\section{Resultados y discusión}

Partiendo de los resultados de la investigación sobre concepciones de libertad, ya desde un comienzo la noción de libertad apuntó a dos tendencias: una, la de un poder hacer o un asunto político; la otra, la del anhelo interno que se convierte en diálogo interno. A partir de las dos tendencias expresadas por los jóvenes sobre la libertad, se afirma desde los mismos estudiantes que la libertad exterior (política, social) resulta vacía y termina por fracasar si los individuos no son "interiormente" libres, es decir si no son independientes y autónomos. ¿Para qué vale, por ejemplo, la libertad de expresión si nadie tiene nada verdaderamente original —o al menos "propio"- que comunicar?, expresaba uno de los estudiantes participantes en la investigación. Así, pues, no es posible abstraer mi libertad interior de la libertad de "todos", de la dimensión política de la libertad y de su desarrollo.

Para el investigador y el docente universitario, el acompañamiento a los jóvenes está planteando nuevos retos orientados a ofrecer una formación que debe permitirles desarrollar una identidad ligada a la vida en común o pública y que apunte a la formación para el desarrollo humano integral. Así, el trabajo educativo se convierte en un escenario compartido para interactuar con los demás, anclado en el espacio pero trascendiendo el tiempo. No se trata de la simple vida biológica sino de una forma de vida humana donde al tutor, en su relación con los estudiantes, se le muestre la otra faceta como acompañante de los procesos de desarrollo personal y social de cada uno, factor que, en consideración de la metodología a distancia y desde la virtualidad, desmonta el 
mito de que si no hay encuentro face to face es imposible impactar la vida y los escenarios de actuación de los profesionales en formación.

Nada mejor que recordar una de las expresiones de un joven entrevistado, participante de la investigación, cuando en sus palabras expresaba: "La libertad se evidencia por el grado de responsabilidad que uno adquiere... En la universidad yo pienso que adquirí un grado de responsabilidad más que el que tenía anteriormente, ya es mi responsabilidad, ya por lo menos yo no hago un trabajo por la nota sino porque yo sé que eso aporta a mi formación como profesional, entonces ya es como el grado de madurez con el que tú asumas esos compromisos. Yo pienso que en este momento la libertad ya va muy consolidada con eso de querer alcanzar algo, con lo de los logros, lo de las metas, y es como tratar también de llevar esa libertad a mi proyecto de vida, $y$ que esa libertad que yo maneje lo haga de una manera bien manejada, y que me permita alcanzar esas metas y lograr lo que me propongo."

Lo anterior se encuentra muy cercano a lo que surge también en la investigación sobre innovaciones curriculares, cuando al abordar el trabajo independiente se alcanza a percibir el impacto que este tipo de actividades pedagógicas puede generar para la construcción de subjetividad y la generación de procesos de autonomía propios de la metodología a distancia, donde el docente tutor cumple con la responsabilidad ya no sólo de acercar al saber de una disciplina para su dominio, sino también de que cada persona (el estudiante tutorado) descubra en esta interacción posibilidades de autorrealización que en términos de Maslow se convierte en el objetivo central de toda la intervención que se propicie dirigida al ser humano.

El estudiante que ingresa "su docente-tutor" a su red social de amigos pretende vincular sus prácticas en el ejercicio de la libertad de la cotidianidad a la vida académica institucional, resignificando el contenido que la universidad le ofrece en términos de lo que debe aprender. Allí el planteamiento vigotskiano de que todo aprendizaje es social actualiza el paradigma educativo según el cual la construcción colectiva del conocimiento ya no es privilegio de un grupo de clase sino de una comunidad de aprendizaje basada en el aporte significativo de sus miembros, donde el vínculo no sólo es académico sino también social y en muchos casos, para el aprendizaje vital, aprender a vivir y a convivir. Así la mediación didáctica o tecnológica es la excusa para el encuentro entre tutores y tutorados en una dinámica multidireccional.

Cualquier diseño instruccional tendrá que vérselas con estas nuevas formas de relación educativa. Así quedó claro cuando la asesora del Ministerio de Educación Nacional, en una de las sesiones desde donde acompañaba el proyecto de transformación de programas a distancia a virtuales, concluía con el mensaje de que "no se trata simplemente de diseñar unos formatos para depositar unos contenidos". Esta reflexión se constituye en un reto para 
los docentes sobre la necesidad de ampliar las fronteras de lo meramente instrumental hacia encuentros face to face de otras calidades y condiciones donde el asunto de la orientación educativa, tal como ha sido definida por autores como Müller(1997) y Ortega (2005), se convierte en un componente intrínseco de la función docente.

Es aquí donde se hace necesario introducir una sucinta descripción de la figura del tutor, quien ha sido observado básicamente en su función académica sin consideraciones acerca del impacto que su presencia, accionar, palabra y estilo tiene sobre sus tutorados. Una de las conclusiones de la investigación sobre concepciones de libertad se dirige precisamente a proponer un acompañamiento más cercano del docente al estudiante, puesto que la experiencia de libertad no se construye, ni tampoco su concepto, de manera aislada, pues su referente es básicamente social, es decir, se es libre para y se es libre de. En ese proceso el estudiante de hoy demanda la presencia de un acompañante que contribuya a formar criterios en torno a las vivencias que puede suscitar la práctica de la libertad; ese acompañante, significativo por la misión encomendada y por su formación, a veces supuesta o esperada, es el docente en su función tutorial.

\section{Conclusiones}

La orientación educativa se ha convertido en un requisito inherente a la función docente, más por solicitud de los mismos estudiantes que por otras consideraciones de orden pedagógico o didáctico. La gran tarea y reto que se postula, en consecuencia, es la necesidad de formar al docente tutor, cualquiera que sea su escenario de actuación, en habilidades para acompañar desde la docencia el desarrollo personal, social y vital de los tutorados, además de las que nacen de su función derivada del vínculo laboral como regente de una asignatura o de la academia.

Esta formación se habrá de dirigir hacia temas como el reconocimiento de las diferentes manifestaciones culturales de quienes son o serán sus tutorados y conocer los estilos de aprendizaje según una caracterización que incluya las diferencias y las variables que los puedan afectar. Muy especialmente debe hacer énfasis en la calidad del acompañamiento grupal e individual, la objetividad y la sensibilidad, con criterio y empatía, para que se generen motivaciones intrínsecas que contribuyan a la construcción de autonomía y resiliencia ante las múltiples condiciones que habrá de sortear el tutorado.

Finalmente, sirva para cerrar esta reflexión y dejar abierta la puerta para otras discusiones sobre el tema, la expresión de Viktor Frankl: "Lo que importa no es que la vida esté cargada de placer o de dolor, sino que esté llena de sentido". 


\section{Arturo de Jesús Madrigal Gil}

Tutoría y orientación educativa:

la otra cara del acompañamiento en la educación a distancia, artículo de reflexión

\section{Referencias bibliográficas}

Beck, U. (2002). Hijos de la libertad. México: Fondo de Cultura Económica.

Betancur, N., Madrigal, A., Cano, N., Palacio, N. Viaje al centro de la libertad [Tesis]. Medellín: CINDE-Universidad de Manizales, 2007.

Bogdan, R. \& Taylor, S. (1976). American Psychologist, vol.31, núm. 1, p. 47.

Económica. Vigotski, L. (2005). Psicología pedagógica. Buenos Aires: Aique.

Garavito, H., Madrigal, A., Palacio, J. \& Urrego, A. Lineamientos pedagógicos y diseño instruccional para el E-Learning. Documento entregado al Ministerio de Educación Nacional en el marco del Proyecto "Transformación de programas de la modalidad a distancia a virtual”. Medellín: Politécnico Colombiano Jaime Isaza Cadavid, 2009.

Garavito, H., Madrigal, A., \& Urrego, A. Innovaciones curriculares para la Facultad de Educación Física, Recreación y Deporte del Politécnico Colombiano Jaime Isaza Cadavid. Investigación en curso para los periodos 2009-2010.

Ghiso, A. (2004). Palabras y presencias adolescentes y juveniles en la universidad. Medellín: CD-R, Universidad de Antioquia.

Maslow, A. (1998). El hombre autorrealizado. Barcelona: Kairós.

Maturana, H. (1996). La persona humana y su crecimiento. Pamplona: Eunsa.

MÜLLER, M. (2000). Docentes tutores. Buenos Aires: Bonum.

ORTEGA, R. (2005). Psicología de la enseñanza y desarrollo de personas y comunidades. México: Fondo de Cultura Económica.

Sen, A. (2000). Desarrollo y libertad. Barcelona: Planeta.

Taylor, C. (1998). Las fuentes del Yo. Barcelona: Paidós.

Tourine, A. (2001). ¿Podremos vivir juntos? México: Fondo de Cultura. 